\title{
Gender Impact on Electrophysiological Activity of the Brain
}

\author{
J. LANGROVÁ ${ }^{1}$, J. KREMLÁČEK ${ }^{1}$, M. KUBA ${ }^{1}$, Z. KUBOVÁ ${ }^{1}$, J. SZANYI $^{1}$ \\ ${ }^{1}$ Department of Pathophysiology, Faculty of Medicine in Hradec Králové, Charles University \\ in Prague, Hradec Králové, Czech Republic
}

Received March 19, 2012

Accepted July 2, 2012

\begin{abstract}
Summary
Gender is presumed to be one of the factors causing interindividual variability in the brain's electrophysiological parameters. Our aim was to characterize the role of gender in visual evoked potentials (VEPs), event-related potentials (ERPs), visual mismatch negativity (VMMN) and the spectral characteristics of the EEG. We examined 42 healthy volunteers ( 21 women and 21 men, aged 20-29 years). We measured VEPs in response to pattern-reversal and motion-onset stimulation, ERPs in an oddball paradigm and VMMN in response to a combination of motion directions presented in the visual periphery. P100 peak latency for $40^{\prime}$ reversal VEPs was significantly shorter in women than in men as determined using a non-parametric Wilcoxon signed-rank test. In addition, women showed higher relative EEG spectral power in the alpha band $(p=0.023)$ and lower power in the theta band $(p=0.004)$. Our results in this small but homogeneous group of subjects confirm previously reported gender influences on pattern-reversal VEPs and the EEG frequency spectrum. Gender should be taken into consideration in establishing norms on these measures. We found no statistically significant differences between women and men for any of the other stimuli presented.
\end{abstract}

\section{Key words}

Gender - Pattern-reversal VEPs - Motion VEPs - Event related potentials $\bullet$ Visual mismatch negativity

\section{Corresponding author}

Jana Langrová, Department of Pathophysiology, Charles University in Prague, Faculty of Medicine in Hradec Králové, Šimkova 870, 50038 Hradec Králové, Czech Republic. E-mail: langrovaj@lfhk.cuni.cz

\section{Introduction}

Visual evoked potentials (VEPs) are used to interrogate the visual pathway from the retina up through high-level visual cortices. These responses can be measured noninvasively and at low cost. The stimulus most commonly used in VEP acquisition is a luminancereversing high contrast checkerboard, which predominantly activates the primary visual cortex (V1) (Seki et al. 1996, Brecelj et al. 1998). Pattern-reversal VEPs (P-VEPs) P100 peak latency and amplitude depend heavily on the pattern's contrast and the visual acuity of the tested subjects (Kubova et al. 1995). Onset of motion in the visual field activates the dorsal visual stream and evokes motion-onset VEPs (M-VEPs), which are relatively independent of contrast and visual acuity (Kubova et al. 1995). Detection of event-related potentials (ERPs) such as the visual mismatch negativity (vMMN) or the oddball P300 is an important step toward obtaining insight into higher-order cognitive functions. Combining information from these various types of visual evoked potentials can extend our understanding of brain function and elucidate the causes of many diseases that affect the central nervous system (CNS).

Gender is presumed to be one of the factors causing interindividual variation in the electrophysiological parameters of the human brain. Many studies have examined gender effects on P-VEPs (e.g., Fenwick et al. 1981, Allison et al. 1984, Cohn et al. 1985, Malcolm et al. 2002, Gregori et al. 2006) and, more recently, on ERPs (Polich and Kok 1995, Hoffman and Polich 1999, Sangal and Sangal 1996, Steffensen et al. 2008).

The effect of gender on P-VEP parameters is inconsistent across studies. While some studies have 
found the dominant P100 peak to be larger in girls than boys (Snyder et al. 1981, Allison et al. 1984, Cohn et al. 1985, Emmerson-Hanover et al. 1994), and many believe that this gender difference continues into adulthood (Allison et al. 1984, Celesia et al. 1987, EmmersonHanover et al. 1994), other authors have found that PVEP peak amplitude does not differ across gender (Snyder et al. 1981, Cohn et al. 1985). An analogous effect has also been reported for P100 peak latency, with some authors reporting shorter latencies in women (Fenwick et al. 1981, Emmerson-Hanover et al. 1994, Malcolm et al. 2002, Gregori et al. 2006) and others reporting no gender difference (Cohn et al. 1985, Mitchell et al. 1987). Celesia et al. (1987) found that the effect of gender on P100 latencies depends on the size of the pattern displayed. Although the effect of gender was obvious for smaller checks (15' of visual angle), no difference between males and females was observed for larger checks (30').

A similar gender difference has also been shown in ERPs. Hoffman and Polich (1999) reported that the P300 peak showed larger amplitude and shorter latency in women than in men. However, Steffensen et al. (2008) only found a smaller P300 peak amplitude in men, and Sangal and Sangal (1996) reported no gender difference in P300 latency or amplitude.

The distribution of EEG power across the lifespan also differs across genders. The markers of EEG maturation are decreases in slow wave activity (theta, delta) and increases in faster activity (alpha, beta) (Somsen et al. 1997, Martinović et al. 1998, Clarke et al. 2001, Segalowitz et al. 2010, Cragg et al. 2011). Clarke et al. (2001) found that girls' EEG matured more slowly than boys' during childhood, but during adolescence the difference was eliminated. However, Gasser et al. (1988) found no gender differences in the EEG power spectrum, likely because of high interindividual variability. The alpha rhythm does not mature until at least age 16 (Marcuse et al. 2008).

If men's and women's electrophysiological responses differ, pooling these data inflates the variability of the parameter estimates, thus negatively influencing the sensitivity of clinical examinations.

With this study, we sought to contribute to the estimation of the gender effect on visual information processing at different levels and on spontaneous brain activity and to assess the importance of that effect for clinical examinations of individual subjects.

\section{Methods}

\section{Subjects}

We examined 21 pairs of age-matched healthy individuals (university students) aged 20-29 years. Our subjects had no ophthalmological or neurological disease. Informed consent was obtained from each subject after the test procedure had been explained to him or her. The study was approved by the Ethics Committee, University Hospital Hradec Králové.

\section{VEPS}

\section{Pattern-reversal VEPS (P-VEPs)}

We used a checkerboard with high luminance contrast (96\% according to Michelson's formula), reversing at $2 \mathrm{~Hz}$, with check sizes of 10', 20' and 40'.

\section{Motion-onset VEPS (M-VEPs)}

Two types of moving stimuli were used:

a) Translational linear motion of isolated checks (M-VEPs L) (check size 40', check-to-check distance 120 ' on both vertical and horizontal axes, moving at velocity $10 \mathrm{deg} / \mathrm{s}$ ) with direction of motion randomly ordered to reduce adaptation of direction-specific cortical neurons.

b) Radial motion of sine-wave modulated concentric circles, contracting and expanding at random, with decreasing spatial frequency (1-0.2 c/deg) toward the periphery of the visual field to account for cortical magnification and with increasing motion velocity (5-25 $\mathrm{deg} / \mathrm{s}$ ) toward the periphery to account for different motion sensitivities in the center versus the periphery of the visual field. We recorded three types of VEPs to radial motion-onset:

- M-VEPs to full field stimulus subtending 37×28 deg (M-VEPs FF),

- M-VEPs to central 8 deg of the visual field (M-VEPs $\mathrm{c} 8^{\circ}$ ),

- M-VEPs to periphery of the stimulus field outside the central 20 deg (M-VEPs $\left.\mathrm{m} 20^{\circ}\right)$.

All moving stimuli were moved for $200 \mathrm{~ms}$ followed by a stationary period of $1000 \mathrm{~ms}$.

VEPs to binocularly observed stimuli were computed by averaging 40 single responses, each of which consisted of a 440-ms epoch sampled at $500 \mathrm{~Hz}$. In P-VEPs we measured peak latency and absolute mean inter-peak amplitude (P100-(N75+N145)/2) of the main P100 peak. For M-VEPs, we measured peak latency and the absolute mean inter-peak amplitude 
$((\mathrm{P} 1+\mathrm{P} 2) / 2-\mathrm{N} 160)$ of the N160 peak, which has been shown to represent the main motion-specific component of this VEP type (Kuba and Kubova 1992, Heinrich
2007, Kuba et al. 2007). The variables represent absolute peak amplitudes of the respective VEPs as they are shown in the Figure 1.

\section{Pattern-Reversal VEPs}

\section{Motion-Onset VEPs}
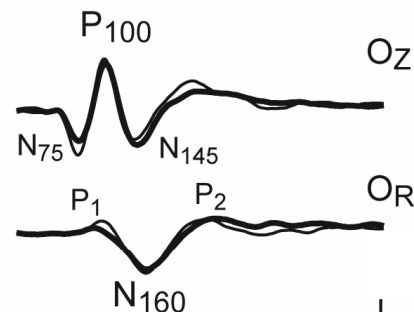

$\mathrm{O}_{\mathrm{R}}$ $10 \mu \mathrm{V}$

$100 \mathrm{~ms}$

$$
\begin{array}{r}
\text { vMMN }- \text { males }(n=21) \\
- \text { females }(n=21)
\end{array}
$$

$$
\text { ODD-BALL - males }(n=21)
$$$$
\text { - females }(n=21)
$$
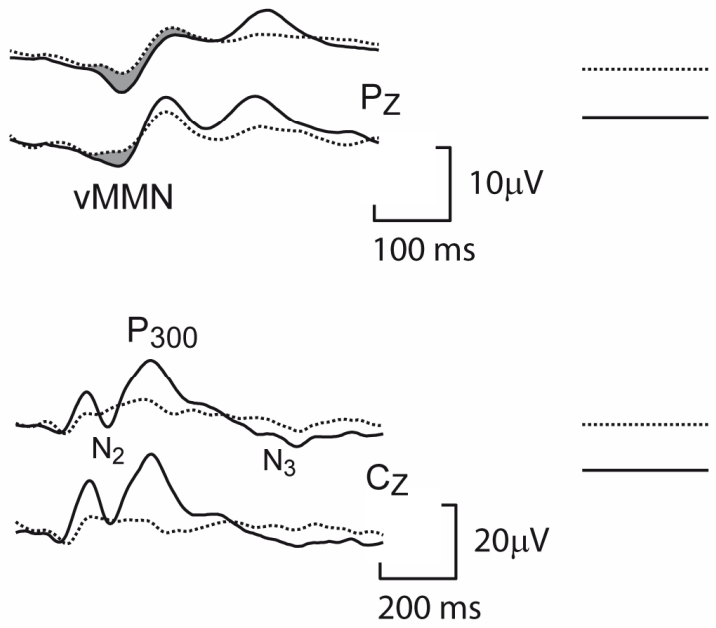

MALES $(n=21)$

FEMALES $(n=21)$

FREQUENT, NON-TARGET RARE, TARGET

Fig. 1. Obtained VEPs/ERPs. The first row shows P-VEPs. The longer latency of the P100 peak (see Table 1a) in men (thick line) compared to women (thin line) is statistically significant but barely observable. The N160 peak, characteristic of the response to motiononset stimuli, did not show any statistically significant gender difference, as depicted in the second row. Each group's responses to the VMMN paradigm (standard ERP - dotted line; deviant ERP - solid line) overlap in the middle of the figure. The grey color represents AUC, which did not differ between groups. The bottom part of the figure depicts ERPs recorded during the visual odd-ball paradigm. These responses show similar overlap to the VMMN responses. Responses to frequent non-target stimuli are plotted with a dotted line; responses to rare targets are plotted with a solid line. The groups did not differ in latency or amplitude of the characteristic peak P300. All plotted VEPs and ERPs are total averages over the specified group.

We used the $\mathrm{O}_{\mathrm{Z}}$ derivation (the derivation with maximum response and the lowest variability) to evaluate P-VEP parameters. Because the generation of M-VEPs is known to differ across individuals (Holliday et al. 1998, Kremlacek et al. 1998), we selected the optimal derivation for each subject. The optimal derivation was the one with the shortest N160 peak latency, unless the latencies were not significantly different, in which case it was the derivation with largest amplitude. For each subject, we chose an optimal derivation from the $\mathrm{O}_{L}, \mathrm{O}_{\mathrm{R}}$, $\mathrm{O}_{Z}$ and $\mathrm{P}_{Z}$ derivations.

Each VEP was acquired twice, and mean values for each subject were submitted to the following statistical analysis.

\section{Event related potentials - ERPs}

Binocular ERPs were recorded during an oddball test, in which the letter X (a frequent non-target stimulus appearing with $75 \%$ probability) and Arabic digits 1-9 (rare target stimuli appearing with $25 \%$ probability) appeared in a pseudorandomly intermixed sequence. Both $\mathrm{X}$ and the Arabic digits were presented in white at the center of the black stimulus field (average luminance of entire field $1 \mathrm{~cd} / \mathrm{m}^{2}$ ) and subtended $5.7 \times 6.3 \mathrm{deg}$ of the visual angle. Each stimulus was displayed for $500 \mathrm{~ms}$ and followed by a black screen with a fixation point for $500 \mathrm{~ms}$. To calculate ERPs, 20 epochs to target stimuli and 20 randomly selected epochs to non-target stimuli (both of 1000 ms duration with sampling frequency of $250 \mathrm{~Hz}$ ) were averaged for each condition. Before averaging, the epochs with artifacts (primarily blinks) were manually rejected. In the ERPs to frequent and rare stimuli, the absolute mean inter-peak amplitudes (P300$(\mathrm{N} 2+\mathrm{N} 3) / 2)$ and peak latencies of the P300 were 
measured using the central $\left(\mathrm{C}_{\mathrm{Z}}\right)$ derivation.

\section{Visual mismatch negativity $-v M M N$}

The test paradigm was specifically designed to elicit the vMMN and was based on the three-stimulus design used by from Tales et al. (1999). There were three motion-onset events:

- standard condition (88\%) - upward motion of a horizontal sinusoidal grating with low (10\%) contrast, spatial frequency of $0.1 \mathrm{c} / \mathrm{deg}$ and velocity of $50 \mathrm{deg} / \mathrm{s}$, presented outside the central $15 \mathrm{deg}$ of the visual field;

- deviant condition (6 \%) - downward motion otherwise identical to the standard condition; and

- rare condition (6\%) - a horizontal sinusoidal grating with low (10\%) contrast, spatial frequency of $1 \mathrm{c} / \mathrm{deg}$ and velocity of $5 \mathrm{deg} / \mathrm{s}$, presented inside the central $5 \mathrm{deg}$ of the visual field. The motion duration was $200 \mathrm{~ms}$, and the stationary pattern was presented for $600 \mathrm{~ms}$.

The vMMN was evaluated using area under the curve (AUC), computed as the integral of the difference between ERPs to standard versus deviant stimuli. The AUC approached zero for similar ERP responses to standard and deviant stimuli and had negative values when ERPs to deviant stimuli were relatively more negative.

All stimuli were presented on a 21 inch computer monitor (Vision Master Pro 510, Iiyama, Japan) subtending a $37 \times 28$ deg of visual angle at $0.6 \mathrm{~m}$ viewing distance. The monitor was driven using the Visual Stimulus Generator 2/5 (CRS Ltd., UK) at a vertical refresh frequency of $105 \mathrm{~Hz}$. A mean luminance of $17 \mathrm{~cd} / \mathrm{m}^{2}$ was used for all VEP, ERP and vMMN stimuli. Electrophysiological acquisition was performed in a darkened, sound-attenuated, electromagnetically shielded room with a background luminance of $0.1 \mathrm{~cd} / \mathrm{m}^{2}$. During the experiment, the subjects sat in a comfortable dental chair with a neck support to reduce muscle artifacts. Correct fixation on the center of the stimulus field was monitored via infrared charge-coupled device camera.

Detailed stimulus parameters have been previously described for the radial motion stimuli (Kremlacek 2004 et al.) and for the mismatch negativity stimuli (Kremlacek 2006 et al.).

Spontaneous electroencephalographic activity - EEG

For the EEG spectral analysis, we recorded $64 \mathrm{~s}$ of resting EEG with subjects' eyes closed. To estimate the power spectral density of the recording, we averaged 16 periodograms computed using a Fourier Transformation in 4-s EEG segments using a rectangular window. To estimate of the relative power of the EEG spectrum, the following parameters were calculated: the delta $(1.75-4 \mathrm{~Hz})$, theta $(4.25-8 \mathrm{~Hz})$, alpha $(8.25-12 \mathrm{~Hz})$, beta1 $(12.25-20 \mathrm{~Hz})$, and beta2 $(20.25-30 \mathrm{~Hz})$ bands; the theta/alpha relative power ratio; and the frequency of the dominant peak.

\section{Recordings}

Pseudo-unipolar recordings were acquired from the midline $\left(\mathrm{O}_{\mathrm{Z}}, \mathrm{P}_{\mathrm{Z}}, \mathrm{C}_{\mathrm{Z}}\right.$ and $\left.\mathrm{F}_{\mathrm{Z}}\right)$ and lateral occipital $\left(\mathrm{O}_{\mathrm{L}}\right.$ $\left(\mathrm{O}_{\mathrm{R}}\right) 5 \mathrm{~cm}$ to the left (right) from the $\mathrm{O}_{Z}$ position) derivations with the right earlobe reference.

The signal was amplified by a factor of 20,000 (Contact Precision Instruments, PSYLAB, System 5, UK) in the frequency band of $0.3-100 \mathrm{~Hz}$ at $-3 \mathrm{~dB}$ with a rolloff of 6 and $12 \mathrm{~dB}$ per octave. After amplification, the signal was sampled at $500 \mathrm{~Hz}$ for VEPs, $250 \mathrm{~Hz}$ for ERPs and $100 \mathrm{~Hz}$ for spontaneous EEG. The VEP/ERP recordings were synchronized with the backward trace of the monitor's electron beam immediately before the first video frame of a given stimulus.

Analysis

Because the measured values were not normally distributed, a non-parametric Wilcoxon signed-rank test was applied to assess significant differences.

\section{Results}

In a previous study, we found that subject age substantially influenced VEP parameters (Langrova et al. 2006). In this study, we tried to minimize this effect by examining healthy volunteers within a narrow age range.

We found that the latency of the main component (P100) for P-VEPs with element size 40' was shorter in women than in men $(p=0.012)$. The P100 peak amplitude was not affected by gender. We found no significant differences in P-VEPs to patterns with other element sizes (P-VEPs 20', 10'). We also found no gender differences in peak latency or amplitude of the main component (N160) evoked by motion-onset stimulation. Table 1 summarizes the median, $25^{\text {th }}$ and $75^{\text {th }}$ percentiles of latencies (a) and amplitudes (b) of P100 peaks of P-VEPs, N160 peak of M-VEPs, and statistical results from the corresponding derivation. 
Table 1. The effects of gender on the latency $[\mathrm{ms}]$ and amplitude $[\mu \mathrm{V}]$ of all tested VEPs obtained at the optimal derivation. Values are expressed as median, $25^{\text {th }}$ and $75^{\text {th }}$ percentiles. Asterisks represent significance level $(* p<0.05)$.

a) VEPs latency $[\mathrm{ms}]$

\begin{tabular}{|c|c|c|c|c|c|}
\hline & $\begin{array}{l}\text { Evaluated } \\
\text { parameter }\end{array}$ & Males & Females & p-level & $\begin{array}{c}\text { Significance } \\
\text { level }\end{array}$ \\
\hline P-VEPs $40^{\prime}$ & $\mathrm{P} 100$ & $109(107: 114)$ & $108(104: 110)$ & 0.012 & $*$ \\
\hline$P$-VEPS $20^{\prime}$ & $\mathrm{P} 100$ & $110(107: 113)$ & $108(107: 109)$ & 0.365 & n.s. \\
\hline$P$-VEPs $10^{\prime}$ & $\mathrm{P} 100$ & $119(112: 120)$ & $117(114: 118)$ & 0.748 & n.s. \\
\hline$M-V E P S L$ & N160 & $157(148: 162)$ & $151(148: 161)$ & 0.487 & n.s. \\
\hline$M-V E P s F F$ & N160 & $148(143: 154)$ & $156(136: 161)$ & 0.664 & n.s. \\
\hline$M-V E P S m 20^{\circ}$ & N160 & $152(146: 162)$ & $154(150: 158)$ & 0.768 & n.s. \\
\hline$M-V E P S c 8^{\circ}$ & N160 & $152(148: 158)$ & 153 (136:159) & 0.465 & n.s. \\
\hline ERP target & P300 & $368(336: 376)$ & $372(364: 380)$ & 0.087 & n.s. \\
\hline
\end{tabular}

b) VEPs amplitude $[\mu \mathrm{V}]$

\begin{tabular}{|c|c|c|c|c|c|}
\hline & $\begin{array}{l}\text { Evaluated } \\
\text { parameter }\end{array}$ & Males & Females & p-level & $\begin{array}{c}\text { Significance } \\
\text { level }\end{array}$ \\
\hline$P-V E P S 40^{\prime}$ & $\mathrm{P} 100$ & $11.5(10.3: 14.6)$ & $15.0(11.2: 16.1)$ & 0.305 & n.s. \\
\hline$P$-VEPs $20^{\prime}$ & P100 & $10.7(9.1: 14.6)$ & $14.2(12.0: 16.6)$ & 0.067 & n.s. \\
\hline$P$-VEPS $10^{\prime}$ & P100 & $13.1(10.3: 16.1)$ & $16.0(11.8: 19.1)$ & 0.181 & n.s. \\
\hline$M-V E P S L$ & N160 & $6.8(5.1: 8.3)$ & $7.7(5.4: 10.2)$ & 0.322 & n.s. \\
\hline$M-V E P s F F$ & N160 & $10.4(9.0: 11.4)$ & $10.4(7.7: 12.4)$ & 0.958 & n.s. \\
\hline$M$-VEPs $m 20^{\circ}$ & N160 & $9.8(7.5: 10.9)$ & $9.2(7.6: 10.6)$ & 0.614 & n.s. \\
\hline$M-V E P S c 8^{\circ}$ & N160 & $9.5(8.3: 11.8)$ & $10.3(8.9: 12.0)$ & 0.889 & n.s. \\
\hline$v M M N$ 120-240 ms [ $\left.\mu V^{*} m s\right]$ & AUC & $8.9(-53.8: 24.6)$ & $-30.2(-53.6: 78.8)$ & 0.217 & n.s. \\
\hline ERP target & P300 & $17.0(14.5: 22.6)$ & $17.8(13.2: 23.2)$ & 0.852 & n.s. \\
\hline
\end{tabular}

Table 2. Effects of gender on relative spectral EEG power [\%] and frequency of the EEG spectrum dominant peak [Hz]. Values are expressed as median, $25^{\text {th }}$ and $75^{\text {th }}$ percentiles. Asterisks represent significance level $(* p<0.05 ; * * p<0.01)$.

\begin{tabular}{|c|c|c|c|c|}
\hline & Males & Females & p-level & $\begin{array}{c}\text { Significance } \\
\text { level }\end{array}$ \\
\hline Dominant peak frequency [Hz] & $9.5(9.0: 10.3)$ & $10.3(9.8: 10.8)$ & 0.093 & n.s. \\
\hline delta & $12.0(10.1: 16.6)$ & $9.5(8.0: 10.9)$ & 0.073 & n.s. \\
\hline theta & $16.4(13.6: 22.2)$ & $11.9(9.6: 16.2)$ & 0.004 & $* *$ \\
\hline alpha & $51.6(39.1: 61.4)$ & $60.7(54.6: 67.3)$ & 0.023 & $*$ \\
\hline betal & $9.4(6.7: 12.8)$ & $9.3(7.7: 14.5)$ & 0.872 & n.s. \\
\hline beta2 & $4.7(3.1: 6.3)$ & $6.1(3.8: 7.5)$ & 0.538 & n.s. \\
\hline theta/alpha coefficient & $0.30(0.23: 0.51)$ & $0.21(0.15: 0.29)$ & 0.007 & $* *$ \\
\hline
\end{tabular}

There was no difference between males and females in the P300 component of ERPs to the rare condition. Table 1a and $1 \mathrm{~b}$ contain the P300 latencies and amplitudes, respectively that were determined using the $\mathrm{C}_{\mathrm{Z}}$ derivation.

We visually inspected the total average, pooling 
across genders, of the vMMN to find the interval of maximal difference between responses to the standard and deviant conditions. This interval appeared at 120-240 $\mathrm{ms}$, analogous to the same interval for the auditory MMN. There were no gender differences in AUC over this interval, as assessed by a Wilcoxon test for paired measures (Figure 1).

Significant gender differences were found in the frequency spectrum of the EEG, with a greater power in the alpha band for women $(\mathrm{p}=0.023)$ and in theta band for men $(p=0.004)$. Additionally, the theta/alpha ratio was significantly lower for women than for men $(p=0.007)$; see Table 2. We did not find any difference in the frequency of the dominant peak in alpha power ( $p=0.093$ ). Our quantitative analysis of EEG power is summarized in Table 2.

Figure 1 shows the total averages for the VEPs and ERPs, which reflect different levels of visual information processing. The total of the averages were not used in statistical comparisons, as they obscure the paired structure of the experiment; however, these values are shown for demonstration purposes.

\section{Discussion}

In this study, we investigated whether gender must be taken into account in calculating norms for VEPs (an index of low-level sensory processing), ERPs and vMMN (indices of the cognitive level of visual processing) and spectral characteristics of the EEG.

Most studies have found shorter VEP peak latencies (Fenwick et al. 1981, Malcolm et al. 2002) and larger peak amplitudes (Allison et al. 1984, Kaneda et al. 1996) in women, and various explanations of these findings have been proposed. Physical characteristics, such as head size (Guthkelch et al. 1987, Malcolm et al. 2002, Gregori et al. 2006) and body temperature (Kaneda et al. 1996), are often suggested as sources of gender differences on these measures. It is plausible that head size could affect the latency of the P100 wave. The slight gender difference in P100 latencies corresponds to the slightly smaller average head size in women. Indeed, Gregori et al. (2006) examined men and women with the same skull size and found no statistically significant differences in VEP peak latency. Unfortunately, we did not measure skull size in this study and therefore cannot verify its impact on our results.

In addition to physical considerations, neuroendocrinological and neurological factors, including gonadal steroids, cortisol, thyroxine and $\gamma$-aminobutyric acid (Kaneda et al. 1996, Sannita 2006), may also be relevant. Many studies have found that estrogens, especially $17 \beta$-estradiol, affect brain function throughout the lifespan, ranging from early developmental stages to the aging processes in older adults (Hutchison et al. 1995). Much work has shown positive effects not only on brain perfusion and metabolism but also on neuronal protection (McCullough et al. 2003, Krause et al. 2006, Irwin et al. 2008). However, we have found no reports linking estrogen to the development of visual perception.

In our study, we evaluated a homogeneous group of 42 healthy subjects. We observed very small gender differences, approximately $1 \mathrm{~ms}$, in P-VEP peak latencies, and only P-VEPs 40' showed a statistically significant gender difference in the P100 peak latency. Although we did not measure any of the aforementioned factors confounding gender differences in VEPs, it seems that gender plays a negligible role in early sensory processing of visual information.

Unlike other studies (Fenwick et al. 1981, Malcolm et al. 2002), we found very small sex differences in P100 peak latency. These differences could be caused by the age of our volunteers, as the gender differences for this measure seem to change across the lifespan (Emmerson-Hanover et al. 1994). Although the authors found distinct P100 latency differences in subjects aged 6-20 and 50-80 years, the differences were smaller in the 20-50-year-old age group.

The previously published findings are inconsistent with respect to gender differences in cognitive ERPs. A study by Sangal and Sangal (1996) found no gender difference in the P300 wave, but a more recent study by Hoffman and Polich (1999) reported larger P300 peak amplitudes in women than men. Our result indicating the absence of a gender difference in the ERP to the rare response (i.e., the P300 wave) supports the previous finding. This inconsistency in the investigations of gender differences in cognitive ERPs might be partially explainable by differences in the stimulus design and the experimental procedure.

Our most striking gender differences appeared in the spectral characteristics of spontaneous EEG. This result is consistent with the results reviewed by Sannita (2006) and might be attributable to testosterone levels, which correlate positively with theta EEG activity. In both men and women, only a small proportion of the EEG power appeared in the lower frequencies (delta, theta); most of the EEG power was present at higher 
frequencies (alpha). This finding represents a marker of maturation in the EEG (Somsen et al. 1997, Clarke et al. 2001, Segalowitz et al. 2010, Cragg et al. 2011). Kaneda et al. (1996) found significantly larger theta and smaller alpha2 powers in women, which is the opposite of our findings. Possible reasons for this discrepancy might be the substantially larger age range and a different method of EEG recording.

The aforementioned relationship between the degree of maturity and distribution of the EEG power spectrum may explain the sex difference we observed in the alpha and theta activity. Matousek (1968) found that the best indicator of maturity is the ratio of theta and alpha activity. In our sample, women showed a higher level of maturity as quantified by this ratio.

In a large sample (almost 1,500 subjects), Chiang et al. (2011) showed statistically significant nonlinear changes in alpha power across the lifespan that differed between men and women. Men showed higher alpha power that decreased steeply until age 20. In women, a smaller, consistent decrease in alpha power was observed throughout the lifespan. The ages of our subjects fall in the period in which Chiang et al. (2011) found a higher alpha power in women compared to men, and this result was recapitulated in our data.

Our results demonstrate various gender differences in the electrophysiological parameters of human brain function. Although there are negligible gender differences for motion-onset VEPs, ERPs or vMMN, obviating the need to calculate separate norms for men and women, P-VEPs and the EEG frequency spectrum seem to be more influenced by gender, and separate gender-dependent norms should be constructed for these measures.
A limitation of this study should be mentioned in the context of our negative results. If gender represents a factor with strong impact on some electrophysiological marker (as in P-VEPs 40', where we find a $1 \mathrm{~ms}$ difference between groups and a narrow interquartile range), then we can make valid conclusions about gender differences in that marker using a sample of 42 subjects. However, a null result for an electrophysiological marker with a higher variability, such as cognitive ERPs, indicates merely that any gender difference in these parameters was not strong enough to be detected in our sample. To explore a small effect of size approximately 0.2 (Cohen 1992), such as the implicit latency of the P300 peak (estimate based on our intergroup difference and variability), we require a sample size of approximately 200 subjects to ensure the statistical significance of the results. Among studies exploring gender effects on electrophysiological parameters, our sample is larger than some and smaller than others (Fenwick et al. 1981 - 48 subjects; Celesia et al. 1987 112 subjects; Guthkelch et al. 1987 - 16 subjects; Emmerson-Hanover et al. 1994 - 406 subjects; Kaneda et al. 1996 - 200 subjects; Gregori et al. 2006 - 54 subjects; Malcolm et al. 2002 - 52 subjects; Steffensen et al. 2008 -30 subjects; Cragg et al. $2011-56$ subjects).

\section{Conflict of Interest}

There is no conflict of interest.

\section{Acknowledgements}

This work was supported by Grant Agency of the Czech Republic 309/09/0869 and by the programme P37/07 (PRVOUK).

\section{References}

ALLISON T, HUME AL, WOOD CC, GOFF WR: Developmental and aging changes in somatosensory, auditory and visual evoked potentials. Electroencephalogr Clin Neurophysiol 58: 14-24, 1984.

BRECELJ J, KAKIGI R, KOYAMA S, HOSHIYAMA M: Visual evoked magnetic responses to central and peripheral stimulation: simultaneous VEP recordings. Brain Topogr 10: 227-237, 1998.

CELESIA GG, KAUFMAN D, CONE S: Effects of age and sex on pattern electroretinograms and visual evoked potentials. Electroencephalogr Clin Neurophysiol 68: 161-171, 1987.

CHIANG AK, RENNIE CJ, ROBINSON PA, VAN ALBADA SJ, KERR CC: Age trends and sex differences of alpha rhythms including split alpha peaks. Clin Neurophysiol 122: 1505-1517, 2011.

CLARKE AR, BARRY RJ, MCCARTHY R, SELIKOWITZ M: Age and sex effects in the EEG: development of the normal child. Clin Neurophysiol 112: 806-814, 2001.

COHEN J: A power primer. Psychol Bull 112: 155-159, 1992. 
COHN NB, KIRCHER J, EMMERSON RY, DUSTMAN RE: Pattern reversal evoked potentials: age, sex and hemispheric asymmetry. Electroencephalogr Clin Neurophysiol 62: 399-405, 1985.

CRAGG L, KOVACEVIC N, MCINTOSH AR, POULSEN C, MARTINU K, LEONARD G, PAUS T: Maturation of EEG power spectra in early adolescence: a longitudinal study. Dev Sci 14: 935-943, 2011.

EMMERSON-HANOVER R, SHEARER DE, CREEL DJ, DUSTMAN RE: Pattern reversal evoked potentials: gender differences and age-related changes in amplitude and latency. Electroencephalogr Clin Neurophysiol 92: 93$101,1994$.

FENWICK PB, BROWN D, HENNESEY J: The visual evoked response to pattern reversal in 'normal' 6-11-year-old children. Electroencephalogr Clin Neurophysiol 51: 49-62, 1981.

GASSER T, VERLEGER R, BÄCHER P, SROKA L: Development of the EEG of school-age children and adolescents. I. Analysis of band power. Electroencephalogr Clin Neurophysiol 69: 91-99, 1988.

GREGORI B, PRO S, BOMBELli F, LA RICCIA M, ACCORNERO N: Vep latency: sex and head size. Clin Neurophysiol 117: 1154-1157, 2006.

GUTHKELCH AN, BURSICK D, SCLABASSI RJ: The relationship of the latency of the visual P100 wave to gender and head size. Electroencephalogr Clin Neurophysiol 68: 219-222, 1987.

HEINRICH SP: A primer on motion visual evoked potentials. Doc Ophthalmol 14: 83-105, 2007.

HOFFMAN LD, POLICH J: P300, handedness, and corpus callosal size: gender, modality, and task. Int $J$ Psychophysiol 31: 163-174, 1999.

HOLLIDAY IE, MEESE TS, BARNES GR: Evoked magnetic fields to optic flow stimuli are largest for expansion. Perception (Suppl) 27: 17, 1998.

HUTCHISON JB, BEYER C, HUTCHISON RE, WOZNIAK A: Sexual dimorphism in the developmental regulation of brain aromatase. J Steroid Biochem Mol Biol 53: 307-313, 1995.

IRWIN RW, YAO J, HAMILTON RT, CADENAS E, BRITON RD, NILSEN J: Progesterone and estrogen regulate oxidative metabolism in brain mitochondria. Endocrinology 149: 3167-3175, 2008.

KANEDA Y, NAKAYAMA H, KAGAWA K, FURUTA N, IKUTA T: Sex differences in visual evoked potential and electroencephalogram of healthy adults. Tokushima J Exp Med 43: 143-157, 1996.

KRAUSE DN, DUCKLES SP, PELLIGRINO DA: Influence of sex steroid hormones on cerebrovascular function. J Appl Physiol 101: 1252-1261, 2006.

KREMLACEK J, KUBA M, KUBOVA Z: Electrophysiological manifestation of first-order motion perception. Perception (Suppl) 27: 192-193, 1998.

KREMLACEK J, KUBA M, KUBOVA Z, CHLUBNOVA J: Motion-onset VEPs to translating, radial, rotating and spiral stimuli. Doc Ophthalmol 109: 169-175, 2004.

KREMLACEK J, KUBA M, KUBOVA Z, LANGROVA J: Visual mismatch negativity elicited by magnocellular system activation. Vision Res 46: 485-490, 2006.

KUBA M, KUBOVA Z: Visual evoked potentials specific for motion onset. Doc Ophthalmol 80: 83-89, 1992.

KUBA M, KUBOVA Z, KREMLACEK J, LANGROVA J: Motion-onset VEPs: characteristics, methods and diagnostic use. Vision Res 47: 189-202, 2007.

KUBOVA Z, KUBA M, SPEKREIJSE H, BLAKEMORE C: Contrast dependence of motion-onset and pattern-reversal evoked potentials. Vision Res 35: 197-205, 1995.

LANGROVA J, KUBA M, KREMLACEK J, KUBOVA Z, VIT F: Motion-onset VEPs reflect long maturation and early aging of visual motion-processing system. Vision Res 46: 536-544, 2006.

MALCOLM CA, MCCULLOCH DL, SHEPHERD AJ: Pattern-reversal visual evoked potentials in infants: gender differences during early visual maturation. Dev Med Child Neurol 44: 345-351, 2002.

MARCUSE LV, SCHNEIDER M, MORTATI KA, DONNELLY KM, ARNEDO V, GRANT AC: Quantitative analysis of the EEG posterior-dominant rhythm in healthy adolescents. Clin Neurophysiol 119: 1778-1781, 2008.

MARTINOVIĆ Z, JOVANOVIĆ V, RISTANOVIĆ D: EEG power spectra of normal preadolescent twins. Gender differences of quantitative EEG maturation. Neurophysiol Clin 28: 231-248, 1998.

MATOUSEK M: Frequency analysis in routine electroencephalography. Electroencephalogr Clin Neurophysiol 24: 365-373, 1968. 
MCCULLOUGH LD, BLIZZARD K, SIMPSON ER, OZ OK, HURN PD: Aromatase cytochrome P450 and extragonadal estrogen play a role in ischemic neuroprotection. $J$ Neurosci 23: 8701-8705, 2003.

MITCHELL KW, HOWE JW, SPENCER SR: Visual evoked potentials in the older population: age and gender effects. Clin Phys Physiol Meas 8: 317-324, 1987.

POLICH J, KOK A: Cognitive and biological determinants of P300: an integrative review. Biol Psychol 41: 103-146, 1995.

SANGAL B, SANGAL JM: Topography of auditory and visual P300 in normal adults. Clin Electroencephalogr 27: 145-150, 1996.

SANNITA WG: Individual variability, end-point effects and possible biases in electrophysiological research. Clin Neurophysiol 117: 2569-2583, 2006.

SEGALOWITZ SJ, SANTESSO DL, JETHA MK: Electrophysiological changes during adolescence: a review. Brain Cogn 72: 86-100, 2010.

SEKI K, NAKASATO N, FUJITA S, HATANAKA K, KAWAMURA T, KANNO A, YOSHIMOTO T: Neuromagnetic evidence that the P100 component of the pattern reversal visual evoked response originates in the bottom of the calcarine fissure. Electroencephalogr Clin Neurophysiol 100: 436-442, 1996.

SNYDER EW, DUSTMAN RE, SHEARER DE: Pattern reversal evoked potential amplitudes: life span changes. Electroencephalogr Clin Neurophysiol 52: 429-434, 1981.

SOMSEN RJ, VAN'T KLOOSTER BJ, VAN DER MOLEN MW, VAN LEEUWEN HM, LICHT R: Growth spurts in brain maturation during middle childhood as indexed by EEG power spectra. Biol Psychol 44: 187-209, 1997.

STEFFENSEN SC, OHRAN AJ, SHIPP DN, HALES K, STOBBS SH, FLEMING DE: Gender-selective effects of the P300 and N400 components of the visual evoked potential. Vision Res 48: 917-925, 2008.

TALES A, NEWTON P, TROSCIANKO T, BUTLER S: Mismatch negativity in the visual modality. Neuroreport 10: 3363-3367, 1999. 